\section{Variation in Maximum Counting Rates During Myocardial Blood Flow Quantification Using ${ }^{82}$ Rb PET}

TO THE EDITOR: With great interest, we read the recent article by Renaud et al. entitled "Characterization of 3-Dimensional PET

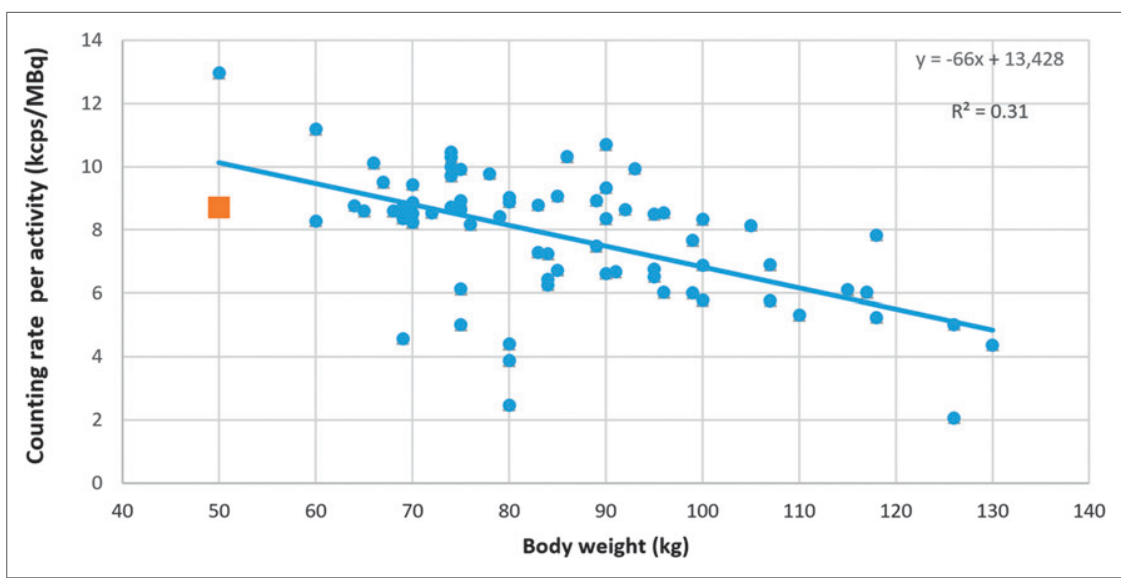

FIGURE 1. Maximum counting rate as encountered in patients (dots) and in phantom (square), normalized to ${ }^{82} \mathrm{Rb}$ tracer activity, as function of body weight. Line represents linear fit. Fit result and coefficient of determination are included in top right corner.

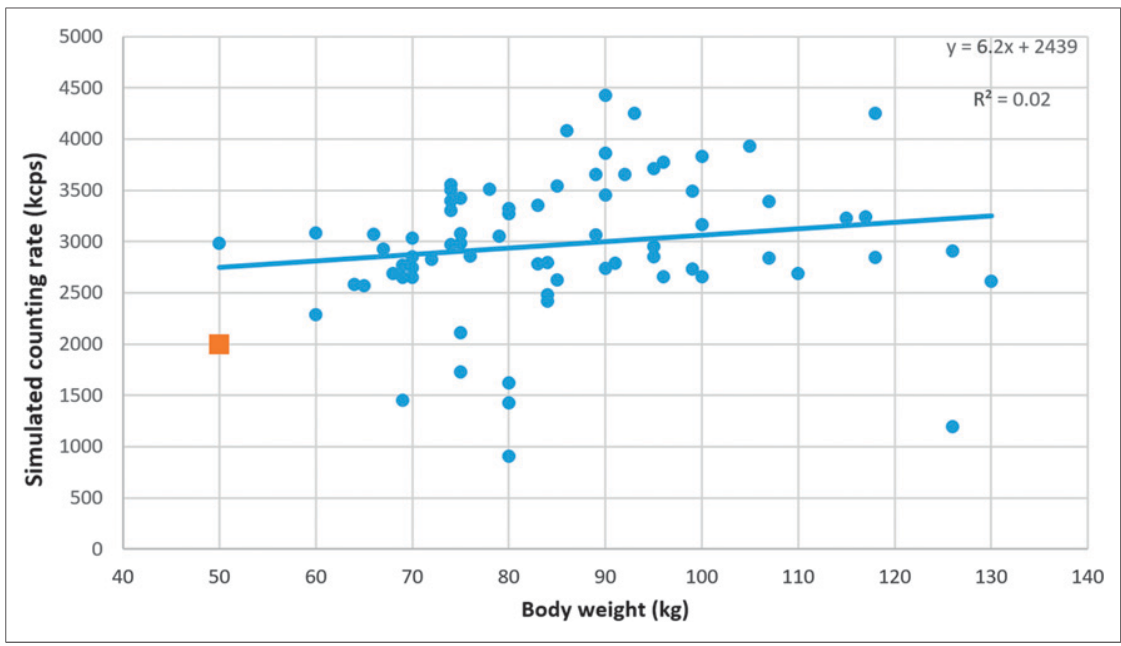

FIGURE 2. Simulated maximum counting rate in patients (dots) and in phantom (square) when $4.6 \mathrm{MBq} / \mathrm{kg}$ of ${ }^{82} \mathrm{Rb}$ was applied as function of body weight. Dashed line represents linear fit. Fit result and coefficient of determination are included in top right corner. Maximum simulated counting rate was higher in $92 \%$ of patients than the phantom result. Furthermore, there was no significant correlation between maximum counting rate and body weight when $4.6 \mathrm{MBq} / \mathrm{kg}$ was applied.
Systems for Accurate Quantification of Myocardial Blood Flow" (1). The authors describe a method to obtain the maximum injected activity for which accurate quantification of myocardial blood flow (MBF) can be achieved. We think that this method majorly conibutes to the existing knowledge. It shows that accurate dyPET systems if maximum injected activities are respected to limit peak dead-time losses during the bolus first-pass transit.

The authors translated the maximum activities for accurate MBF as determined by phantom studies to maximum patientspecific tracer activities (in $\mathrm{MBq} / \mathrm{kg}$ ). However, in our opinion, this translation might be an oversimplified approach, because the activity distribution and photon attenuation in patients during the first-pass transit do not solely depend on body weight. We think that application of the presented method may lead to higher counting rates in some patients than the maximum counting rates as derived from the phantom study. These higher counting rates may subsequently hamper accurate MBF quantification.

To ground our viewpoint, we obtained the maximum prompt coincidence counting rate on our PET system (Ingenuity TF; Philips Healthcare) using the same phantom as described by Renaud et al. (1). Next, we retrospectively obtained the maximum accepted counting rates during myocardial perfusion imaging at rest with ${ }^{82} \mathrm{Rb}$ PET for 72 consecutive patients. All patients provided written informed consent for the use of their data for research purposes. An activity of $740 \mathrm{MBq}$ was injected at a flow of $50 \mathrm{~mL} / \mathrm{min}$ (CardioGen-82; Bracco Diagnostics Inc.) in the phantom and in patients. Next, we studied the effect of using the recommended injected activity per body weight as proposed by Renaud et al. (1). Therefore, we multiplied the measured maximum counting rate, normalized to the injected activity, with body weight and 4.6 MBq/kg for each patient. This way, we obtained a simulated maximum counting rate when using $4.6 \mathrm{MBq} / \mathrm{kg}$. We chose $4.6 \mathrm{MBq} /$ $\mathrm{kg}$ because this is the maximum activity per body weight ensuring accurate quantification for a comparable PET system (Gemini; Philips Healthcare) (1). The body weight equivalent of the phantom was set at $50 \mathrm{~kg}(1)$.

The maximum simulated counting rate for the phantom was 2,001 kcps. The mean body weight of the included patients was $86 \pm 17 \mathrm{~kg}$, and the body mass index was $28.6 \pm 5.4 \mathrm{~kg} / \mathrm{m}^{2}$. We found a mean simulated 
maximum counting rate of $2,975 \pm 681 \mathrm{kcps}$, with a large range of 909-4,431 kcps (Figs. 1 and 2). In 66 patients (92\%), we found a maximum counting rate that was higher than $2,001 \mathrm{kcps}$, the value that we derived from our phantom study. This implies that the suggested translation to $4.6 \mathrm{MBq} / \mathrm{kg}$ may result in counting rates exceeding the $10 \%$ activity bias criteria (1) in $92 \%$ of the patients, possibly leading to biased MBF measurements. Nevertheless, our results support the use of a weight-based activity, because this resulted in simulated maximum counting rates that were independent of body weight.

Applying a lower maximum injected weight-based activity than the one suggested by Renaud et al. (1) can account for the large variation in maximum counting rates encountered in patients and may prevent biased MBF measurements. To ensure accurate MBF quantification with ${ }^{82} \mathrm{Rb}$ PET across all patients, we therefore suggest the inclusion of a correction in the translation of phantom results to the maximum weight-based activity in patients.

\section{REFERENCE}

1. Renaud JM, Yip K, Guimond J, et al. Characterization of 3-dimensional PET systems for accurate quantification of myocardial blood flow. J Nucl Med. 2017;58:103-109.

Joris D. van Dijk*

Pieter L. Jager

Jochen A.C. van Osch

Jorn A. van Dalen

*Isala Hospital

P.O. Box 10400

8000 GK Zwolle, The Netherlands.

E-mail: jorisvdijk@gmail.com

Published online Jan. 19, 2017.

DOI: 10.2967/jnumed.116.187757
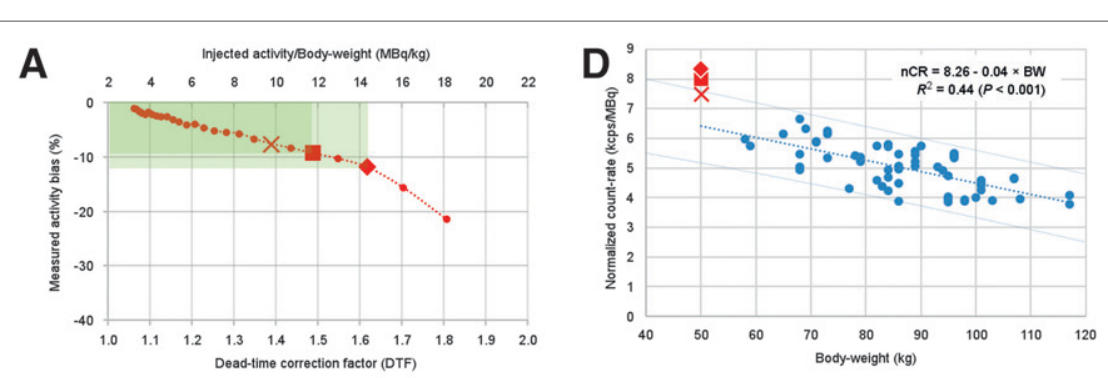

B
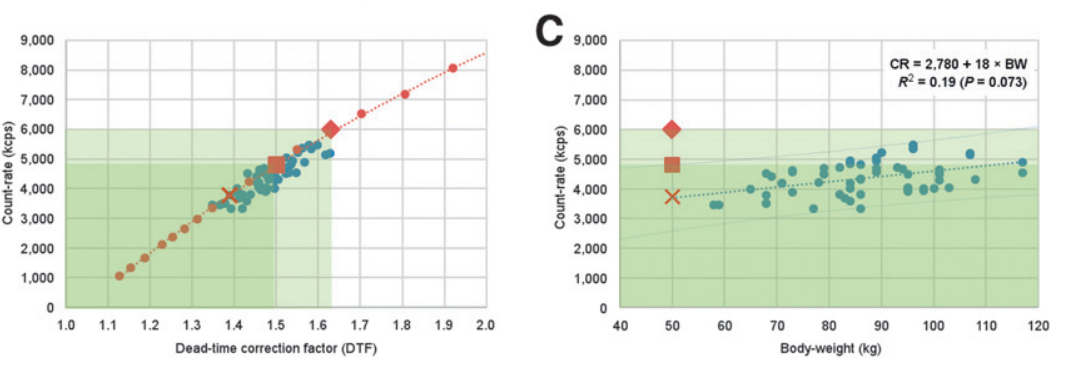

FIGURE 1. (A) Activity bias in decaying phantom study is $<10 \%-12 \%$ (square and diamond markers and green shaded areas) with DTFs $<1.5-1.6$. (B) Prompt CRs are closely related to the DTF values in both phantom (red) and patient (blue) studies. (C) Fifty-kilogram weight-equivalent phantom study ( $\mathrm{red} X$ ) is consistent with peak measured patient CRs using $10 \mathrm{MBq} / \mathrm{kg}$ weight-based dosing. (D) Normalized CRs (nCRs) per MBq injected activity decrease with body weight (BW); therefore, smaller patients will have higher peak CRs and higher potential bias when using standard injected activity regardless of body weight.
REPLY: We thank the authors for their interest in our recent paper (1) reporting the dynamic range of current 3-dimensional PET systems and the corresponding weight-based dose limits $(\mathrm{MBq} / \mathrm{kg})$ for accurate measurement of tracer activity and myocardial blood flow (MBF). They suggest that the proposed translation of phantommeasured to patient-recommended dosing may lead to counting rates (CRs) that exceed the recommended limits in some patients. We agree that the cardiac phantom scans are a simplified representation not be used alone without comparison to patient data confirming the weight-based dose limit that allows accurate quantification of $\mathrm{MBF}$ on a particular scanner, as shown in our paper. In this regard the authors are commended for comparing their patient-measured CRs with those obtained using the cardiac torso phantom. However, we note that their phantom-measured peak CR was obtained using a different method than we recommended, which is to determine the instantaneous total activity in the scanner field of view and the corresponding dead-time correction factor (DTF) (or prompt CR) at which quantitative accuracy is maintained with a less than $10 \%$ bias. The measured or simulated phantom CRs reported by van Dijk et al. after injection of their standard dose of $740 \mathrm{MBq}$ do not appear to have any relation to the $10 \%$ bias criteria recommended in our paper. Their phantom data do appear to us to be fully consistent with the spread of patient values around the lines-of-best-fit, alhough prediction bounds were not included in their figures.

To further clarify, our original methodology determined the DTF with a less than $10 \%$ bias in reconstructed activity dence CR (Figure 1B), which can also be used to establish anc confirm the dynamic range for accurate quantification. Comparative data from our Discovery 690 demonstrate a measured activity bias of less than $10 \%-12 \%$ at a CR of less than 5,000-6,000 kcps and DTF of less than 1.5-1.6 for this particular scanner. As illustrated in Figure 1C, our peak patient CRs are in excellent agreement with the instantaneous phantom $\mathrm{CR}$ corresponding to an injected dose of 9-10 MBq/kg (and $<10 \%$ activity bias). This dose used in our clinical practice is slightly lower than the 11.4 $\mathrm{MBq} / \mathrm{kg}$ maximum value listed for the Discovery 690 scanner in the original paper and accounts for the population variability in peak DTF and CR observed due to differences in patient height, body habitus, or other non-weight-based factors as we reported initially. The distribution of CRs in our patient data is similar to that reported by the authors, with a large percentage of patients having higher peak CRs than observed in the phantom scan with equivalent injected dose. However, the authors should not assume that these higher CRs are associated with inaccurate measured activity values, because they did not report the CR (or DTF) limit required to maintain accuracy on their particular scanner. The lower peak CR observed in the 50-kg weight-equivalent phantom and in the smaller patients is a direct consequence of the recommended weight-based dosing. Although there is a clear trend to 